\title{
A comparison study between near roadway measurements and air pollutant dispersion simulations using an improved line source model
}

\author{
R. Briant ${ }^{1}$, C. Seigneur ${ }^{1}$, M. Gadrat ${ }^{2} \&$ C. Bugajny ${ }^{2}$ \\ ${ }^{1}$ CEREA, Joint Research Laboratory, \\ École des Ponts ParisTech / EDF R\&D, Université Paris-Est, France \\ ${ }^{2}$ Centre d'Étude technique de l'Équipement (CETE) Nord Picardie, \\ France
}

\begin{abstract}
Gaussian plume models, which are widely used to model atmospheric dispersion, provide an exact analytical solution for line sources, such as roads, only when the wind direction is perpendicular to the road. Some approximations have been developed to provide an analytical formula for a line source when the wind direction is not perpendicular to the road; however, such formulas lead to some error and the solution diverges when the wind direction is parallel to the road. A novel approach that reduces the error in the line source formula when the wind direction is not perpendicular to the road was recently developed. This model, combined with a Romberg integration to account for the road section width, has then been used to simulate $\mathrm{NO}_{\mathrm{x}}$ concentrations in two large case studies (1371 road sections for the first case study and 100 for the second). $\mathrm{NO}_{2}$, $\mathrm{NO}$ and $\mathrm{O}_{3}$ concentrations are then computed using the photostationary-state approximation. Finally, $\mathrm{NO}_{2}$ concentrations were successfully compared with near-roadway measurements made at various locations in the domain area (224 locations for the first case study and 70 locations for the second). Results obtained with a standard model used for regulatory applications, ADMS, are also presented. Keywords: Gaussian plume model, line source, polyphemus, ADMS.
\end{abstract}




\section{Introduction}

Atmospheric dispersion models are used to estimate the air quality impacts of road traffic emissions for many purposes, such as attainment of ambient air quality standards, health risk assessment and decision support. It may be used for instance to assess the effect of emission control measures or to help select a new road location. It is thus essential to be able to predict with reasonable accuracy the pollutant concentrations associated with vehicle emissions. To that end, analytical models have been developed to simulate the effect of atmospheric dispersion on pollutant concentrations based on an emission rate from a roadway. In open terrain, Gaussian dispersion models are the most commonly used (e.g., [1-4]). Although the Gaussian dispersion formula provides an exact solution to the atmospheric diffusion equation for the dispersion of a pollutant emitted from a point source given some assumptions on stationarity and homogeneity [5], the Gaussian dispersion formula provides an exact solution for the emissions of a pollutant from a line source only in the case where the wind is perpendicular to the line source [6]. It is, therefore, necessary to develop approximations to model atmospheric dispersion from a line source using a Gaussian formulation. One example of such a formulation is that of [7] which reduces the error in the line source formula of [8] when the wind direction is not perpendicular to the road. Although this model performs well for theoretical cases, it has not been evaluated yet with ambient concentration measurements.

Here, we briefly present the model developed in [7] and we combine it with a Romberg integration to simulate the road section width (Section 2). Then in Section 3 we present results of comparison between simulations and measurements. We use this model to simulate $\mathrm{NO}_{\mathrm{x}}$ concentrations in two large case studies (1371 road sections for the first case study and 100 for the second). $\mathrm{NO}_{2}$, NO and $\mathrm{O}_{3}$ concentrations are then computed using the photostationarystate approximation and $\mathrm{NO}_{2}$ concentrations are compared with near-roadway measurements made at various locations of the domain area (224 locations for the first case study and 70 locations for the second).

\section{Gaussian plume model for line sources}

The Gaussian formulation of the concentration field for a pollutant emitted from a line source is the result of the integration of the point source solution over the line source: Equation (1) (reflexion terms are neglected for simplicity).

$$
\mathrm{C}(x, y, z)=\int_{y_{1}}^{y_{2}} \frac{\mathrm{Q}}{2 \pi \mathrm{u} \sigma_{y}(s) \sigma_{z}(s)} \exp \left(\frac{-z^{2}}{2 \sigma_{z}^{2}(s)}-\frac{(y-s)^{2}}{2 \sigma_{y}^{2}(s)}\right) d s
$$

where $\mathrm{C}$ is the pollutant concentration in $g \cdot m^{-3}$ at location $(x, y, z), x$ is the distance from the source along the wind direction in $m, y$ and $z$ are the crosswind distances from the plume centerline in $m, \mathrm{u}$ is the wind velocity in $m . s^{-1}$, $\mathrm{Q}$ is the emission rate in $g . s^{-1}, y_{1}$ and $y_{2}$ the ordinates of the source extremities, 


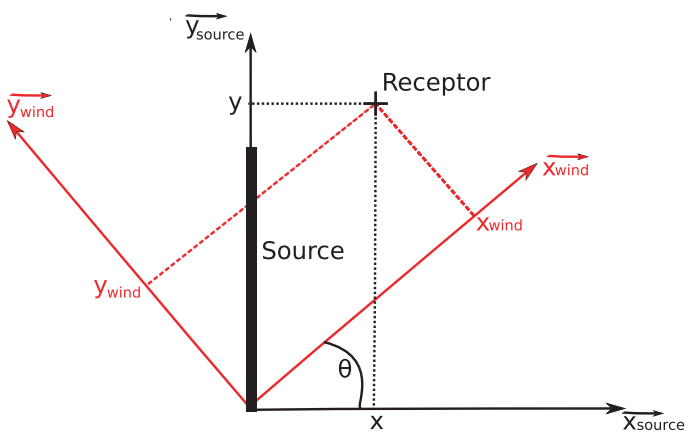

Figure 1: Schematic representation of the source $\left(\vec{x}_{\text {source }}, \vec{y}_{\text {source }}\right)$ and wind $\left(\vec{x}_{\text {wind }}\right.$, $\left.\overrightarrow{\mathrm{y}}_{\text {wind }}\right)$ coordinate systems. The wind angle $\theta$ is the angle between the normal to the source and the wind direction.

and $\sigma_{y}$ and $\sigma_{z}$ are the standard deviations representing pollutant dispersion in the cross-wind directions in $m$, computed here with Briggs's parameterization.

In a perpendicular wind case, both source coordinate system and wind coordinate system are identical (Figure 1). Therefore, the distance of the receptor from the source in the wind direction, needed to compute $\sigma_{y}$ and $\sigma_{z}$, does not change with the integration variable; so no additional approximation is required. For other wind directions, the dependency of standard deviations on the integration variable makes the integration impossible without approximations. Various approximations can be made [6]; we use here a formulation recently proposed by [8].

The Horst-Venkatram (HV) approximation consists in evaluating the integral by approximating the integrand with its behavior near $y_{\text {wind }}=0$ (see Figure 1).

Solving Equation (1) with the HV approximation leads to Equation (2), which provides the concentration field for all wind directions, except $\theta=90^{\circ}$. The term $u \cos \theta$ represents the projection of the wind velocity onto the normal direction to the source. However, when the wind is parallel to the line source $\left(\theta=90^{\circ}\right)$, the term $\cos \theta$, on the denominator of the equation, makes Equation (2) diverge.

$$
\begin{aligned}
& \mathrm{C}(x, y, z)=\frac{\mathrm{Q}}{2 \sqrt{2 \pi} \mathrm{u} \cos \theta \sigma_{z}\left(\mathrm{~d}_{\mathrm{eff}}\right)} \exp \left(\frac{-z^{2}}{2 \sigma_{z}^{2}\left(\mathrm{~d}_{\mathrm{eff}}\right)}\right) \times \\
& {\left[\operatorname{erf}\left(\frac{\left(y-y_{1}\right) \cos \theta-x \sin \theta}{\sqrt{2} \sigma_{y}\left(d_{1}\right)}\right)-\operatorname{erf}\left(\frac{\left(y-y_{2}\right) \cos \theta-x \sin \theta}{\sqrt{2} \sigma_{y}\left(d_{2}\right)}\right)\right]}
\end{aligned}
$$

This solution to the Gaussian equation for a line source has been shown to lead to small acceptable errors compared to an exact solution [8]; nevertheless, some errors remain due to the approximate nature of the solution, especially when the wind is nearly parallel to the line source. In [7] the error made by Equation (2) was computed and parameterized in order to correct the initial formula. For cases where the wind is parallel to the line source, the use of an 
analytical/discretized line source combination, allows one to minimize the error very effectively. Because this combination is only applied for a small range of wind directions, the increase in the overall computational time is not expected to be significant. The objective of this work was to further improve this solution for the concentration field while retaining a computationally-efficient analytical formulation to the extent possible. It provides some improvement in terms of accuracy over previous formulations of the line source Gaussian plume model without being too demanding in terms of computational resources. In addition to what is presented in [7], the model used here also includes a Romberg integration to simulate the road section width. This model was implemented in the modeling platform Polyphemus [9] which is open source and distributed under GNU GPL (http://cerea.enpc.fr/polyphemus) For simplicity, we refer to this new line source model as Polyphemus hereafter.

\section{Comparison to measurements}

The model presented above is evaluated here with actual concentration measurements made by the French technical study and engineering center CETE Nord Picardie. Here we present results of the comparison of simulation results to two cases studies. Those two case studies includes near-roadway air quality estimations measurements by passive tube (to be distinguished from calibrated methods) along with all necessary data required to conduct simulation with Gaussian dispersion models.

\subsection{Case study 1}

This first case study includes concentration measurements made in Paris region, France during winter 2007 and summer 2008. The dataset contains:

- The coordinates of 1371 road sections divided into 5425 smaller, but straight, sections representing a total of $831 \mathrm{~km}$.

- The $\mathrm{NO}_{\mathrm{x}}$ emission rates associated to each road section computed with European model COPERT 3.

- Meteorological data required for a Gaussian model: wind velocity, wind direction, cloud coverage.

- The measured concentrations at 224 receptor points, averaged over each overall time period of the measurement campaign (1 month in winter and 1 month in summer).

- $\mathrm{NO}_{2}$ and $\mathrm{O}_{3}$ background concentrations computed with the Polyphemus Polair3d model [10].

Meteorological data of the specific time period of the measurement campaign were not available. Therefore, for this preliminary study, a generic meteorology of another year was used instead. Although this induces some uncertainty in the results, the use of values averaged over one-month periods minimize the impact of the meteorology. 


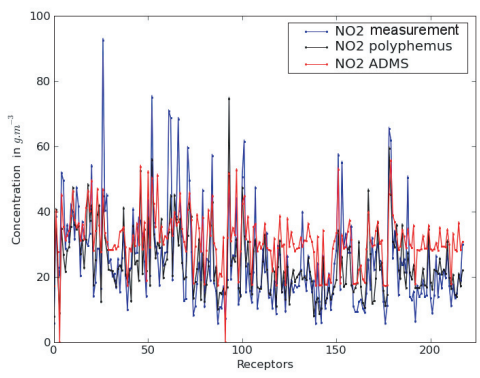

(a) Summer campaign with 'rural' option for Polyphemus.

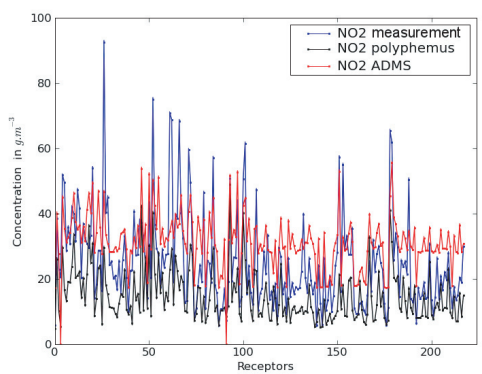

(c) Summer campaign with 'urban' option for Polyphemus.

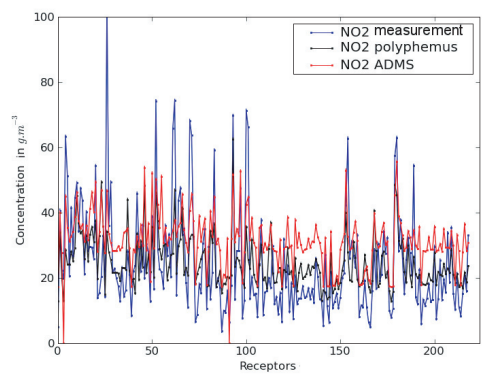

(b) Winter campaign with 'rural' option for Polyphemus.

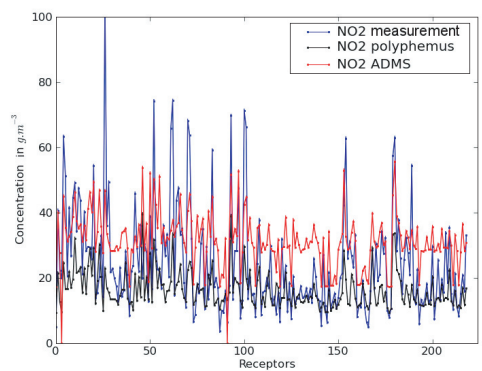

(d) Winter campaign with 'urban' option for Polyphemus.

Figure 2: $\mathrm{NO}_{2}$ concentrations measured and simulated with Polyphemus and ADMS. (note that ADMS results are annual averages rather than periodspecifics values).

The computational time required to simulate a whole month is about 2 to 3 hours with a 2, $4 \mathrm{GHz}$ processor. Moreover, because the meteorological situations are independent, several processors can be used concurrently to decrease the computational burden.

Figure 2 shows comparison results for each of the 224 receptor points. Several indicators were computed to estimate the error made by the model:

- Correlation: $r=\frac{\sum_{i=1}^{N}\left(O_{i}-\bar{O}\right)\left(M_{i}-\bar{M}\right)}{\sum_{i=1}^{N}\left(O_{i}-\bar{O}\right)^{2} \sum_{i=1}^{N}\left(M_{i}-\bar{M}\right)^{2}}$ 
- $R M S E$ (root mean square error): $R M S E=\sqrt{\frac{1}{N} \sum_{i=1}^{N}\left(M_{i}-O_{i}\right)^{2}}$

- $M N E$ (mean normalize error): $M N E=\frac{1}{N} \sum_{i=1}^{N}\left|\frac{M_{i}-O_{i}}{O_{i}}\right|$

- $M N B$ (mean normalize bias): $M N B=\frac{1}{N} \sum_{i=1}^{N} \frac{M_{i}-O_{i}}{O_{i}}$

where $M_{i}$ and $O_{i}$ are the modeled and observed values, respectively.

Both 'rural' and 'urban' land category cases were tested with the Polyphemus model and Table 1 summarizes the results. Polyphemus with the option 'rural' has the best performance for the summer campaign whereas it is with the option 'urban' that performance is the best for the winter campaign (except for the RMSE which is better with the option 'rural'). In addition, it can be seen that results are better in summer than in winter.

Finally, in the last column of Table 1, results, obtain by the CETE Nord Picardie, with the atmospheric dispersion model ADMS [11] are presented. ADMS is a standard Gaussian dispersion model that is widely used for regulatory applications. Polyphemus seems to give better results but it should be noted that results of the ADMS simulation were averaged over a whole year and do not correspond to the time periods of the measurement campaign. That is the reason why the curve of ADMS in Figure 2 is the same in winter and in summer. Nevertheless, this

Table 1: Performance indicators of Polyphemus and ADMS for the case study 1.

\begin{tabular}{|c|c|c|c|}
\hline \multirow{2}{*}{ Summer } & \multicolumn{2}{|c|}{ Polyphemus } & \multirow{2}{*}{ ADMS $^{1}$} \\
\cline { 2 - 3 } & Rural & Urban & \\
\hline Correlation & 0.73 & 0.72 & 0.71 \\
\hline RMSE (in $\mu g \cdot m^{-3}$ ) & 10.08 & 14.77 & 12.03 \\
\hline MNE & 0.30 & 0.39 & 0.56 \\
\hline MNB & 0.09 & -0.36 & 0.47 \\
\hline \hline \multirow{2}{*}{ Winter } & \multicolumn{2}{|c|}{ Polyphemus } & \multirow{2}{*}{ ADMS } \\
\cline { 2 - 3 } & Rural & Urban & \\
\hline Correlation & 0.65 & 0.68 & 0.67 \\
\hline RMSE (in $\mu g \cdot m^{-3}$ ) & 12.43 & 15.12 & 13.94 \\
\hline MNE & 0.48 & 0.34 & 0.72 \\
\hline MNB & 0.28 & -0.15 & 0.62 \\
\hline
\end{tabular}

${ }^{1}$ Note that ADMS results, compared to period-specific values rather than annual average values and, therefore, should be seen as preliminary. 


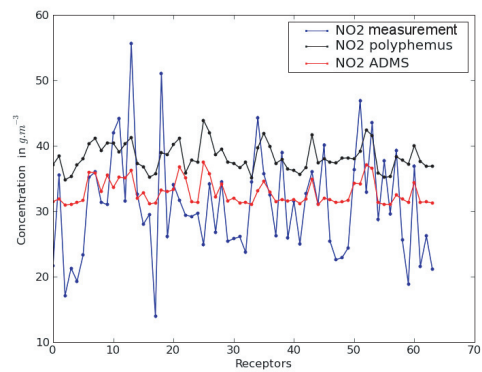

(a) Summer campaign with 'rural' option for Polyphemus.

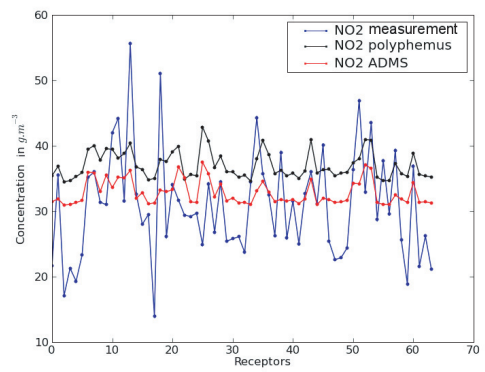

(c) Summer campaign with 'urban' option for Polyphemus.

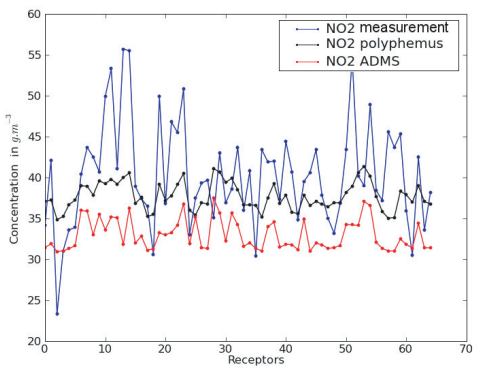

(b) Winter campaign with 'rural' option for Polyphemus.

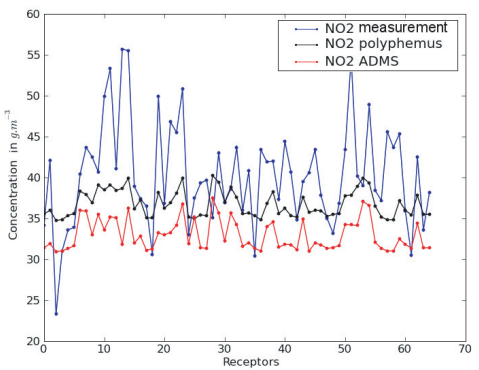

(d) Winter campaign with 'urban' option for Polyphemus.

Figure 3: $\mathrm{NO}_{2}$ concentrations measured and simulated with Polyphemus and ADMS. (note that ADMS results are annual averages rather than periodspecifics values)

results provide some preliminary estimates of the model performance on a large case study.

\subsection{Case study 2}

Measurements were made in the Lille (France) region in 2010 and the dataset contains:

- The coordinates of 100 road sections divided into 362 smaller, but straight, sections representing a total of $29.6 \mathrm{~km}$.

- The $\mathrm{NO}_{\mathrm{x}}$ emission rates associated to each road sections computed with the European model COPERT 4. 
Table 2: Performance indicators of Polyphemus and ADMS for case study 2.

\begin{tabular}{|c|c|c|c|}
\hline \multirow{2}{*}{ Summer } & \multicolumn{2}{|c|}{ Polyphemus } & \multirow{2}{*}{ ADMS $^{2}$} \\
\cline { 2 - 3 } & Rural & Urban & \\
\hline Correlation & 0.47 & 0.54 & 0.51 \\
\hline RMSE (in $\mu g . m^{-3}$ ) & 10.39 & 9.43 & 7.54 \\
\hline MNE & 0.35 & 0.32 & 0.22 \\
\hline MNB & 0.32 & 0.27 & 0.13 \\
\hline \hline Winter & \multicolumn{2}{|c|}{ Polyphemus } & \multirow{2}{*}{ ADMS } \\
\cline { 2 - 3 } & Rural & Urban & \\
\hline Correlation & 0.54 & 0.59 & 0.45 \\
\hline RMSE (in $\mu g . m^{-3}$ ) & 6.15 & 6.54 & 9.27 \\
\hline MNE & 0.11 & 0.12 & 0.18 \\
\hline MNB & -0.04 & -0.07 & -0.16 \\
\hline
\end{tabular}

${ }^{1}$ Note that ADMS results, compared to period-specific values rather than annual average values and, therefore, should be seen as preliminary.

- Meteorological data required for a Gaussian model: wind velocity, wind direction, cloud coverage.

- The measured concentrations at 70 receptor points, average over each overall time period of the measurement campaign (1 month in winter and 1 month in summer).

- $\mathrm{NO}_{2}$ and $\mathrm{O}_{3}$ background concentrations measured at a fixed urban background measurement station.

This case study is much smaller than the previous one and, accordingly, the computational time required for these simulations was much smaller (a few minutes). Nevertheless, this case study presents two advantages over the previous one. First, meteorological data in this case study, match the measurement campaigns time periods. In addition, emission rates were computed with the more recent model COPERT4, instead of COPERT3 in the previous case study.

Figure 3 shows comparison results for each of the 70 receptor points. Performance indicators were computed and are summarize in Table 2. ADMS results, obtain by the CETE Nord Picardie, gives better results for the summer campaign except for the correlation, which is better with Polyphemus with the 'urban' option. For the winter campaign, Polyphemus is better with both 'rural' and 'urban' options.

\section{Conclusion}

The Gaussian plume model of Polyphemus for line sources has been presented and evaluated with two case studies. The first case study a large roadway system, but 
meteorological data did not match measurements time periods. The second case study is smaller but with the correct meteorological data. Polyphemus performs well on both cases when confronted to both measurements and to ADMS model results. Ongoing work is now to incorporate this Gaussian model into a 3D Eulerian gridded model to constitute a plume-in-grid model, which would allow us to improve the representation of the impact of roadway traffic in Eulerian models.

\section{References}

[1] Levitin, J., Härkönen, J., Kukkonen, J. \& Nikmo, J., Evaluation of the caline 4 and car-fmi models against measurements near a major road. Atmos Env, 39, pp. 4439-4452, 2005.

[2] Berger, J., Walker, S.E., Denby, B., Berkowicz, R., Fstrøm, P.L., Ketzel, M., Härkönen, J., Nikmo, J. \& Karppinen, A., Evaluation and inter-comparison of open road line source models currently in use in the nordic countries. Boreal Env Res, 15(319-334), 2010.

[3] Venkatram, A., Isakov, V., Seila, R. \& Baldauf, R., Modeling the impacts of traffic emissions on air toxics concentrations near roadways. Atmos Env, 43, pp. 3191-3199, 2009.

[4] Chen, H., Bai, S., Eisinger, D., Niemeier, D. \& Claggett, M., Predicting nearroad PM2:5 concentrations: comparative assessment of caline4, cal3qhc, and aermod. Transportation Research Record, Journal of the Transportation Research Board, 2123(26-37), 2009.

[5] Csanady, G., Turbulent diffusion in the environment. D Reidel Publishing Company, Dordrecht, The Netherlands, 1973.

[6] Yamartino, R., AIR QUALITY MODELING - Theories, Methodologies, Computational Techniques, and Available Databases and Software. Vol IIISpecial Issues. EnviroComp Institute and the Air \& Waste Management Association, 2008.

[7] Briant, R., Korsakissok, I. \& Seigneur, C., An improved line source model for air pollutant dispersion from roadway traffic. Atmos Env, 2010. In press, doi:10.1016/j.atmosenv.2010.11.016.

[8] Venkatram, A. \& Horst, T., Approximating dispersion from a finite line source. Atmos Env, 40, pp. 2401-2408, 2006.

[9] Mallet, V., Quélo, D., Sportisse, B., Ahmed de Biasi, M., Debry, É., Korsakissok, I., Wu, L., Roustan, Y., Sartelet, K., Tombette, M. \& Foudhil, H., Technical Note: The air quality modeling system Polyphemus. Atmos Chem Phys, 7(20), pp. 5479-5487, 2007.

[10] Roustan, Y., Pausader, M. \& Seigneur, C., Estimating the effect of onroad vehicle emission controls on future air quality in paris, france. Atmos Environ, 2010. In press, doi:10.1016/j.atmosenv.2010.10.010.

[11] McHugh, C., Carruthers, D., Higson, H. \& Dyster, S., Comparison of model evaluation methodologies with application to ADMS 3 and U.S. models. Int J Env Pollut, 16(1-6), 2001. 